J. Dairy Sci. 96:8047-8059

http://dx.doi.org/10.3168/jds.2013-6568

(C) American Dairy Science Association ${ }^{\circledR}, 2013$.

\title{
Development of an automatic cow body condition scoring using body shape signature and Fourier descriptors
}

\author{
A. Bercovich, ${ }^{*}$ Y. Edan, ${ }^{*}$ V. Alchanatis, † U. Moallem,ł Y. Parmet, ${ }^{*}$ H. Honig,‡ E. Maltz,† A. Antler,† \\ and I. Halachmi ${ }^{1}$ \\ *Department of Industrial Engineering and Management, Faculty of Engineering Sciences, Ben-Gurion University of the Negev, \\ Beer-Sheva 84105, Israel \\ †Institute of Agricultural Engineering, and \\ łlnstitute of Animal Science, ARO, The Volcani Center, PO Box 6, Bet Dagan 50250, Israel
}

\section{ABSTRACT}

Body condition evaluation is a common tool to assess energy reserves of dairy cows and to estimate their fatness or thinness. This study presents a computer-vision tool that automatically estimates cow's body condition score. Top-view images of 151 cows were collected on an Israeli research dairy farm using a digital still camera located at the entrance to the milking parlor. The cow's tailhead area and its contour were segmented and extracted automatically. Two types of features of the tailhead contour were extracted: (1) the angles and distances between 5 anatomical points; and (2) the cow signature, which is a 1-dimensional vector of the Euclidean distances from each point in the normalized tailhead contour to the shape center. Two methods were applied to describe the cow's signature and to reduce its dimension: (1) partial least squares regression, and (2) Fourier descriptors of the cow signature. Three prediction models were compared with manual scores of an expert. Results indicate that (1) it is possible to automatically extract and predict body condition from color images without any manual interference; and (2) Fourier descriptors of the cow's signature result in improved performance $\left(\mathrm{R}^{2}=0.77\right)$.

Key words: dairy cow, cow body condition scoring sensor, computer vision, Fourier descriptor

\section{INTRODUCTION}

Body condition scoring estimates a cow's energy reserves and thus fatness or thinness (Hady et al., 1994). Evaluation of BCS is an important management tool for analyzing health problems, feed intake, and optimal time interval between calving and first insemination (Heinrichs and Ishler, 1989). The most popular method

Received January 11, 2013

Accepted August 3, 2013.

${ }^{1}$ Corresponding author: halachmi@volcani.agri.gov.il for evaluating BSC is the 5-point scale (Ferguson et al., 2006: 1 representing emaciated cows and 5 representing obese cows). Currently, BCS is determined manually (Roche et al., 2009). This is a time-consuming task that requires a trained technician. Furthermore, the scores are subjective and may be influenced by previously observed cows (Halachmi et al., 2008). Subjectively assigned scores may not be a perfect indicator of subcutaneous energy reserves.

Despite several attempts to automate body condition scoring of dairy cows (Coffey et al., 2003; Ferguson et al., 2006; Bewley et al., 2008; Halachmi et al., 2008; Azzaro et al., 2011), it is still handled manually with no commercial applications for an automated process. Halachmi et al. (2008) extracted the cow's contour from thermal images. The curvature of the tailhead contour reflected BCS, and this score could be predicted by calculating the mean absolute error between a fitted polynomial and the cow's contour. However, implementation of a thermal camera is relatively expensive and might be problematic in warm environments (Halachmi et al., 2008). Bewley et al. (2008) manually labeled 23 anatomical points on the cow's contour and used the angles created from those points as features for linear regression prediction. Azzaro et al. (2011) used the same 23 points as features for principal component analysis (PCA). However, both of those studies required manual labeling of the 23 anatomical points, which requires time and training and is also not consistent.

Shape signatures such as centroid-contour distances, complex coordinates, or cumulative angle are often used for automatic representations of shapes. These methods do not involve manual labeling, and they are scale-invariant (Zhang and $\mathrm{Lu}, 2004$ ). Zion et al. (2006) described a fish's contours with a polar coordinate signature (distance and angle from each point in the contour to the centroid of the shape) followed by partial least squares (PLS) to compress the data and produce a small number of features for fish type classification. Partial least squares involves selection of 
several latent variables that minimizes the root mean square error in a cross validation scheme, using the "leave-one-out method" (LOOM), a cross-validation statistical estimator of prediction model performance that is frequently used for model selection. The $\mathrm{R}^{2}$ of LOOM is obtained by performing $N$ sample predictions based on the model evaluated from $N-1$ samples (Stone, 1974).

Zhang and $\mathrm{Lu}$ (2004) concluded that Fourier descriptors are an effective tool to describe the variances between signatures of 1-dimensional (1D) centroidcontour distances. Fourier transformation converts any mathematical function to the frequency domain. For $N$ sampling points, the discrete Fourier transform (DFT) yields $N$ Fourier coefficients known as Fourier descriptors (FD). Zhang and $\mathrm{Lu}$ (2004) showed that FD are an efficient tool to overcome sensitivity to noise and difficulties in normalizations. Transformation FD have been successfully applied in the quality inspection of food products, such as potato grading, classification of broccoli heads, and analysis of apple shapes (Brosnan and Sun, 2004). In many cases, only $1 \%$ of the FD are sufficient to describe the essence of a shape's boundary. In addition, FD have physical meaning, they are easy to compute, and they capture both local and global features.

This research builds upon previous studies (Bewley et al., 2008; Halachmi et al., 2008; Azzaro et al., 2011) that aimed to develop an automated computer-vision tool to predict cow BCS using a low-cost digital camera without the need for manual labeling.

\section{MATERIALS AND METHODS}

The main research steps are illustrated in Figure 1.

\section{Image Acquisition}

Data were collected at the Volcani Center ARO experimental farm in Bet Dagan, Israel, between October 2011 and February 2012. The procedures used were approved by the Volcani Center Animal Care Committee. A Nikon D7000 DSLR camera (Nikon Inc., Tokyo, Japan) was located at the milking parlor entrance gate, $2.5 \mathrm{~m}$ above ground and aimed downward. All images were taken before the noon milking. The camera was activated from a personal computer (Camera Control pro 2 software, Nikon Inc.) each time a cow entered through the milking parlor gate and acquired 6 consecutive images with a $1,632 \times 2,464$ resolution. All images were downloaded to the computer and processed offline. Images for processing were selected using the following criteria: (1) the cow's entire tailhead area was included in the frame; (2) the tailhead area was not touching any iron construction objects or another cow; and (3) the tail and legs were straight. Figure 2 demonstrates 2 selected images (top) and 2 rejected images (bottom).

\section{Training and Testing Sets}

Two data sets were collected separately. A training data set containing 87 different images of 71 Holstein cows was collected between October and December 2011 and was used for the model development. A testing data set containing 64 different images of 41 Holstein

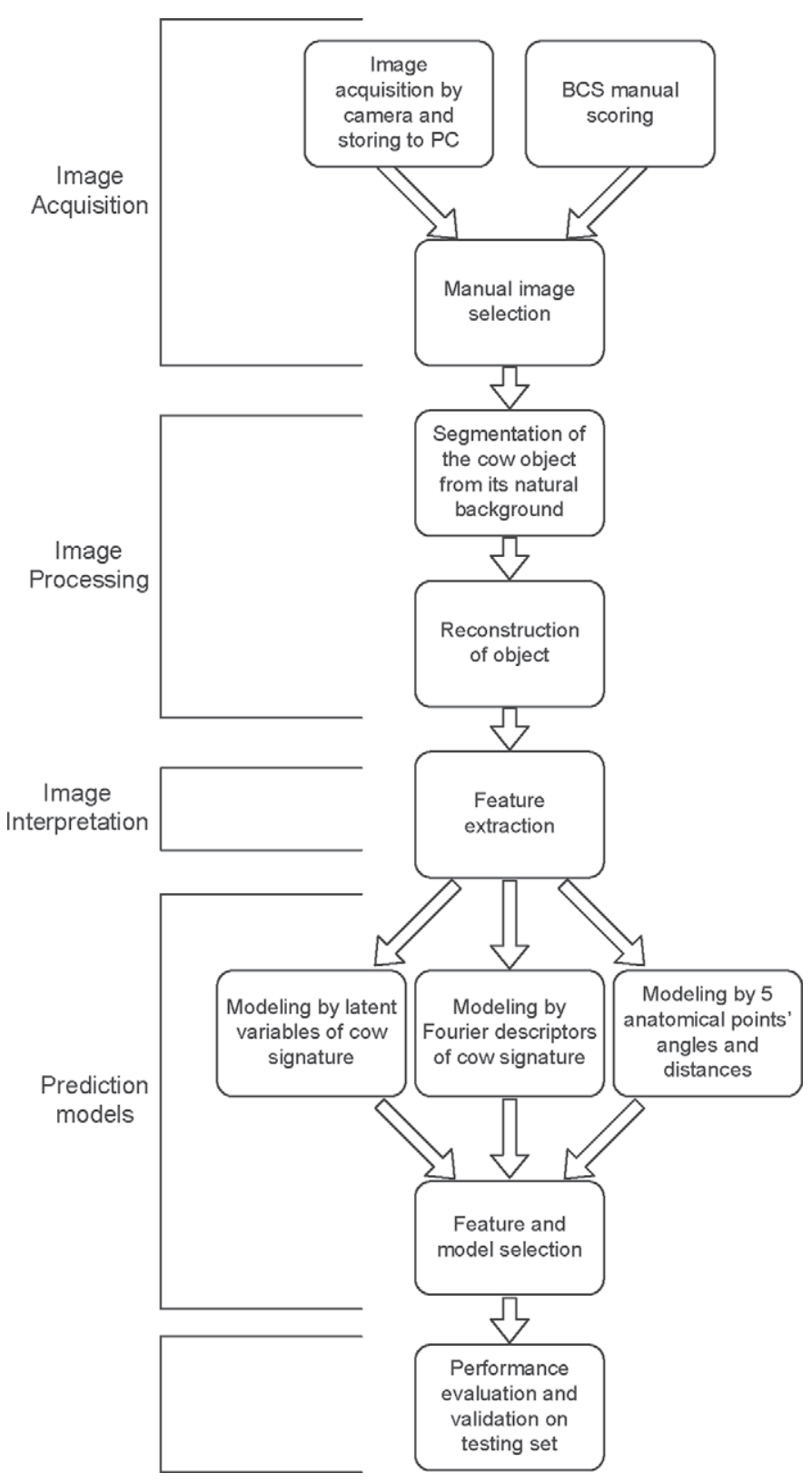

Figure 1. The main research steps. 

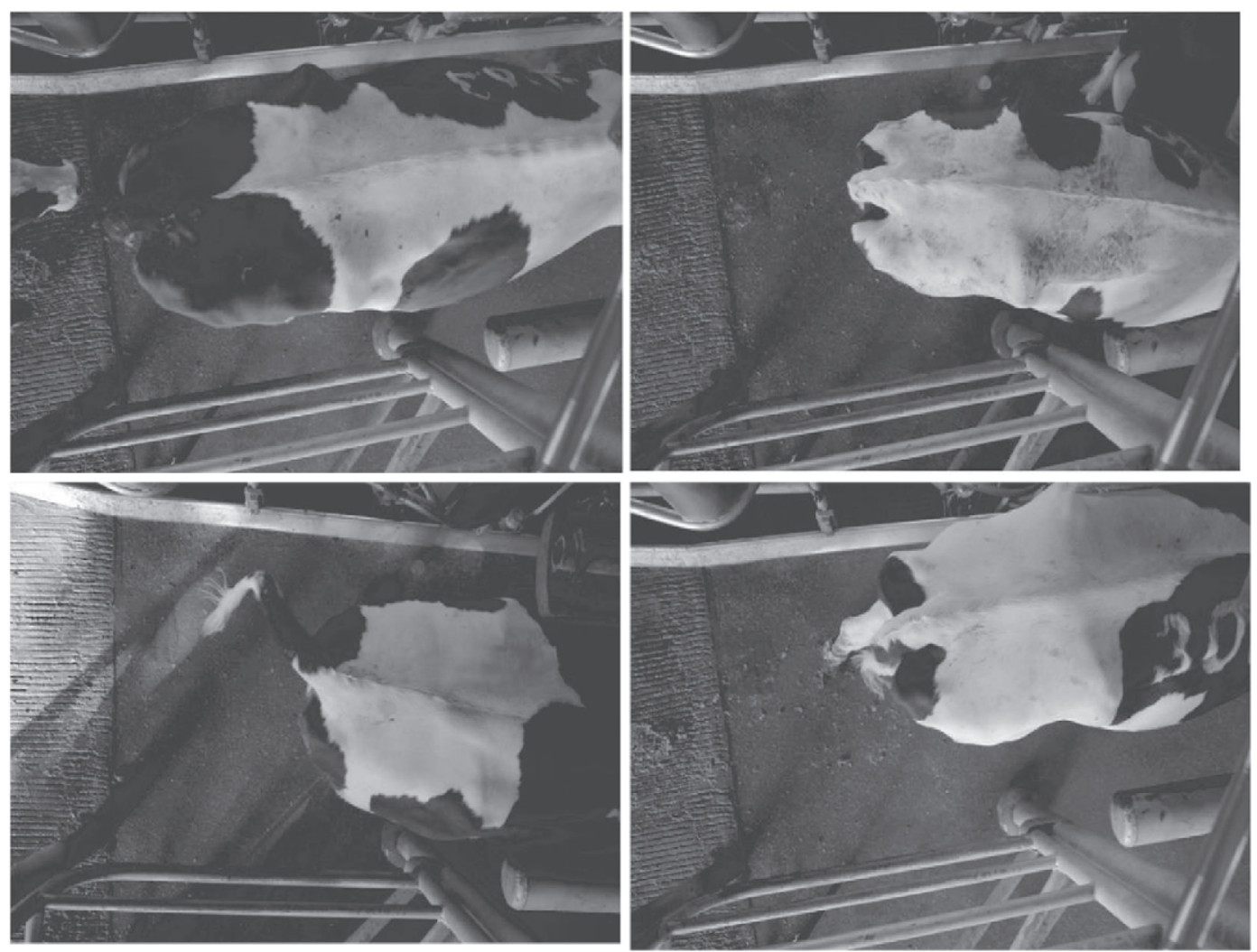

Figure 2. Cows entering the milking parlor. Two selected images (top) and 2 rejected images (bottom). On the bottom left, the tail was not straight. On the bottom right, the cow's left back leg was not straight under the cow.

cows was collected in February 2012 and was used for model validation. All cows were manually scored by an expert using the BCS 1-5 scale (Ferguson et al., 1994) on the day of image acquisition. The BCS expert was a researcher (U. Moallem) who has spent a professional lifetime on a variety of BCS issues. To assess the accuracy level of the BCS reference, the BCS of 42 cows was determined by the BCS expert 3 times within $10 \mathrm{~d}$, assuming that BCS would not change during this short period. The average standard error in this test was 0.07 BCS unit. In our case, more than $95 \%$ of differences were less than 2 standard deviations (SD). The definition of a repeatability coefficient adopted by the British Standards Institution (BSI, 1975) is that $95 \%$ of differences should be less than 2 SD. The BCS evaluation was based on the tailhead and pin bones, but also on the hook bones and the line between the hook and pin bones. The BCS was determined primarily by touching and feeling the pin bones and tailhead, and assessing the cavity between these 2 points. In addition, it was based on evaluating the side view of the shape of the line between pin bones and the hooks and the sharpness of the hooks. In most of the cases, a rear view on the short ribs and the vertebrae at the middle of the back was also taken.

\section{Image-Processing Algorithm}

To separate the cow from its natural background, several common edge-detection methods were applied; namely Sobel, Prewitt, Roberts (Ziou and Tabbone, 1997) and Canny (Canny, 1986). Qualitative analyses of the results indicated that these methods had difficulty finding the tailhead contour among other edges that represented the cow's black and white coloring. To solve this problem, a color transformation followed by automatic thresholding was applied.

Color-Mask Determination. To simplify the segmentation process, the image was transformed into a 1D image using a color mask that emphasized the differences between the objects in the image (Table 1). The color mask was designed to eliminate the black and white stains and construct a homogeneous distribution of the pixel values representing the cow. Eighteen different color masks were tested (Table 1). The color masks were created by red-green-blue (RGB) color spaces and cylindrical coordinates. Cow color was not homogeneous in any hue-saturation-value (HSV) color masks, and those HSV masks were therefore not applied. To compare the effectiveness of the different masks, oneway ANOVA with repeated measures followed by the 
Table 1. The mean difference between the I mask $(\mathrm{R}-\mathrm{G})$ threshold effectiveness and the other 17 masks is the threshold effectiveness

\begin{tabular}{lc}
\hline Color mask $^{1}$ & $\begin{array}{c}\text { Mean } \\
\text { difference } \\
(\mathrm{I})\end{array}$ \\
\hline $\mathrm{R}$ & $0.1601^{* *}$ \\
$\mathrm{G}$ & $0.0841^{* *}$ \\
$\mathrm{~B}$ & $0.0850^{* *}$ \\
$\mathrm{R} /(\mathrm{R}+\mathrm{G}+\mathrm{B})$ & $0.0231^{* *}$ \\
$\mathrm{G} / \mathrm{R}+\mathrm{G}+\mathrm{B})$ & $0.0602^{* *}$ \\
$\mathrm{~B} /(\mathrm{R}+\mathrm{G}+\mathrm{B})$ & $0.0996^{* *}$ \\
$(\mathrm{R}-\mathrm{G})+(\mathrm{R}-\mathrm{B})$ & $0.0427^{* *}$ \\
$(\mathrm{G}-\mathrm{R})+(\mathrm{G}-\mathrm{B})$ & $0.0182^{* *}$ \\
$(\mathrm{~B}-\mathrm{G})+(\mathrm{B}-\mathrm{R})$ & $0.1339^{* *}$ \\
$\mathrm{~S}$ & $0.0284^{* *}$ \\
$\mathrm{~V}$ & $0.1623^{* *}$ \\
$\mathrm{R}-\mathrm{B}$ & $0.0922^{* *}$ \\
$\mathrm{G}-\mathrm{B}$ & $0.2050^{* *}$ \\
$2 \mathrm{G}-\mathrm{R}-\mathrm{B}$ & $0.0182^{* *}$ \\
$(\mathrm{R}-\mathrm{G}) /(\mathrm{R}+\mathrm{G})$ & $0.0210^{* *}$ \\
$(\mathrm{G}-\mathrm{R}) /(\mathrm{R}+\mathrm{B})$ & $0.0212^{* *}$ \\
$(\mathrm{R}-\mathrm{B}) /(\mathrm{R}+\mathrm{B})$ & $0.0603^{* *}$ \\
\hline
\end{tabular}

${ }^{1} \mathrm{R}, \mathrm{G}$, and $\mathrm{B}$ refer to red, green, and blue in RGB presentation; $\mathrm{S}$ and $\mathrm{V}$ refer to saturation and value in the hue, saturation, and value (HSV) color representation model (hue was not used). The color mask column indicates the math calculations of the corresponding R, G, and B values. ${ }^{2}$ Where $\mathrm{I}=\mathrm{R}-\mathrm{G}$

** Mean difference is significant at the 0.01 level (2-tailed).

least significant difference post hoc test was applied. The dependent variable was the effectiveness measure, and the factor was the 18 masks that were described in Table 1. This analysis was performed on the 63 images.

The $\mathrm{R}-\mathrm{G}$ mask was found to be significantly more effective than the others, with a significantly positive mean difference between $\mathrm{R}-\mathrm{G}$ threshold effectiveness and those achieved with the rest of the color masks (Table 1). To select a suitable color mask, the Otsu method (Otsu, 1979) was used for automatic thresholding of the images (applied with Matlab function Graythresh). The Otsu method is a common method used to automatically perform histogram thresholding by assuming that the image contains 2 classes of pixels (the image pixels histogram has a bimodal distribution) and then calculating the optimum value that separates the 2 classes with minimum variance within classes.

Segmentation and Reconstruction Procedure. The segmentation procedure involved the background and acquired cow images and is described in Figure 3. Each image was transformed to the $\mathrm{R}-\mathrm{G}$ space and then transformed to grayscale values (using Matlab function Mat2gray), followed by increasing the image contrast (Matlab function Imadjust). From these images, the automatic threshold was computed by the Otsu method (Otsu, 1979) and the 2 images were transformed to binary images. Figure 4 shows the pixel-distribution histograms of the original background image in grayscale pixel values (upper left). One can see that for the transformed background image ( $\mathrm{R}-\mathrm{G}$ transform), the pixel value distribution is bimodal (upper right). The high values between 50 and 100 are the pixels representing the red floor of the milking parlor. On the bottom left, the values of the transformed pixels are presented in grayscale (values between 0 and 1) and on the bottom right, the pixel values are presented after increasing the contrast in the image. At this stage, the threshold was computed (0.4 for the example image, Figure 4) and then the background image was subtracted from the cow image. The outcome of this process was a binary image containing the cow and noise objects. At this stage, noise can appear for many reasons, such as secondary objects in the image caused by parts of another cow entering the frame, or by another object in the background such as mud or manure "connected" to the cow object. The cow object in the binary image was determined by assuming that it was the largest object in the image, so that the rest of the objects could be ignored. The cow object was then rotated to a horizontal orientation so the edge of the tailhead could be founded by the minimal coordinate of the object in the horizontal axis. Only the upper third of the object was saved to eliminate artificial cuts created in the segmentation by background objects. The last stage involved a $90^{\circ}$ rotation of the object. The result of this process was a binary image of the tailhead area of the cow.

\section{Feature Extraction}

The tailhead contour was extracted from the $x$ and $y$ coordinates of the boundaries in the tailhead binary image. The features extracted from the tailhead contour were (1) the distances and angles between 5 anatomical points. Those 5 points are part of the 23 anatomical points defined by Bewley et al. (2008) as point numbers $9,10,12,14$, and 15 . We defined $\mathrm{X} 1$ to $\mathrm{X} 5$ as the horizontal distances between the points, and Y1 to Y5 as the vertical distances between those points; Angles 1 to 5 were defined as the angles created from those points; (2) the entire tailhead contour was presented as a $1 \mathrm{D}$ vector ("cow signature"), where each point in the contour was presented by its Euclidean distance from the centroid of the shape. Figure 5 illustrates the extracted feature: the original image (a), the binary tailhead area (b), the tailhead contour (c), the cow signature (d), the vertical (Y1..Y 5 ) and horizontal (X1...X5) distance between the anatomical points (e), and the angles (Angle1...Angle5) created from those points (f).

\section{Feature-Extraction Algorithm}

The tailhead contour was normalized, by first sorting the vertical axis coordinates and then selecting the high- 
a
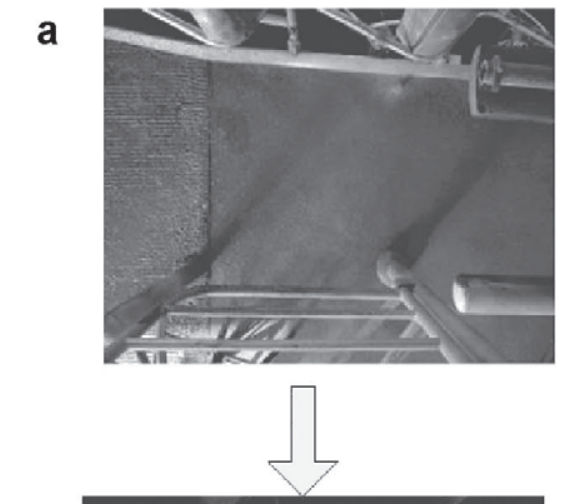

b

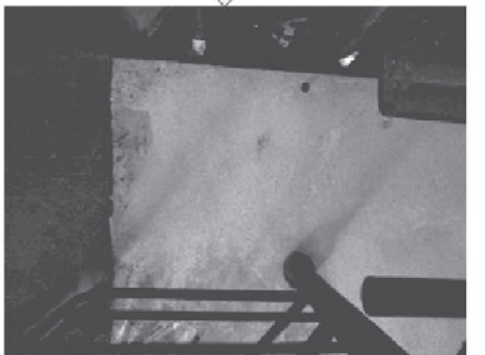

C
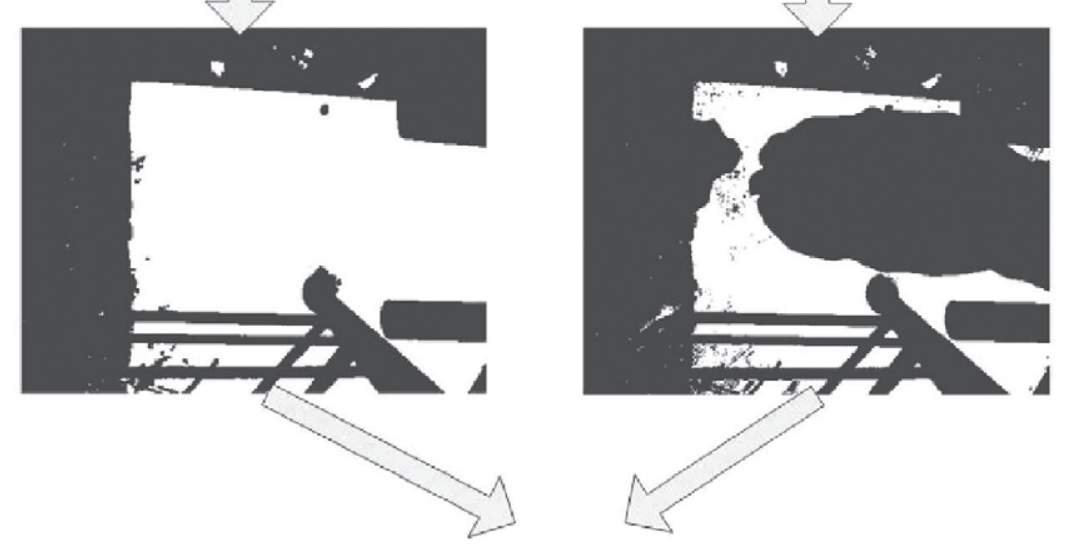

d
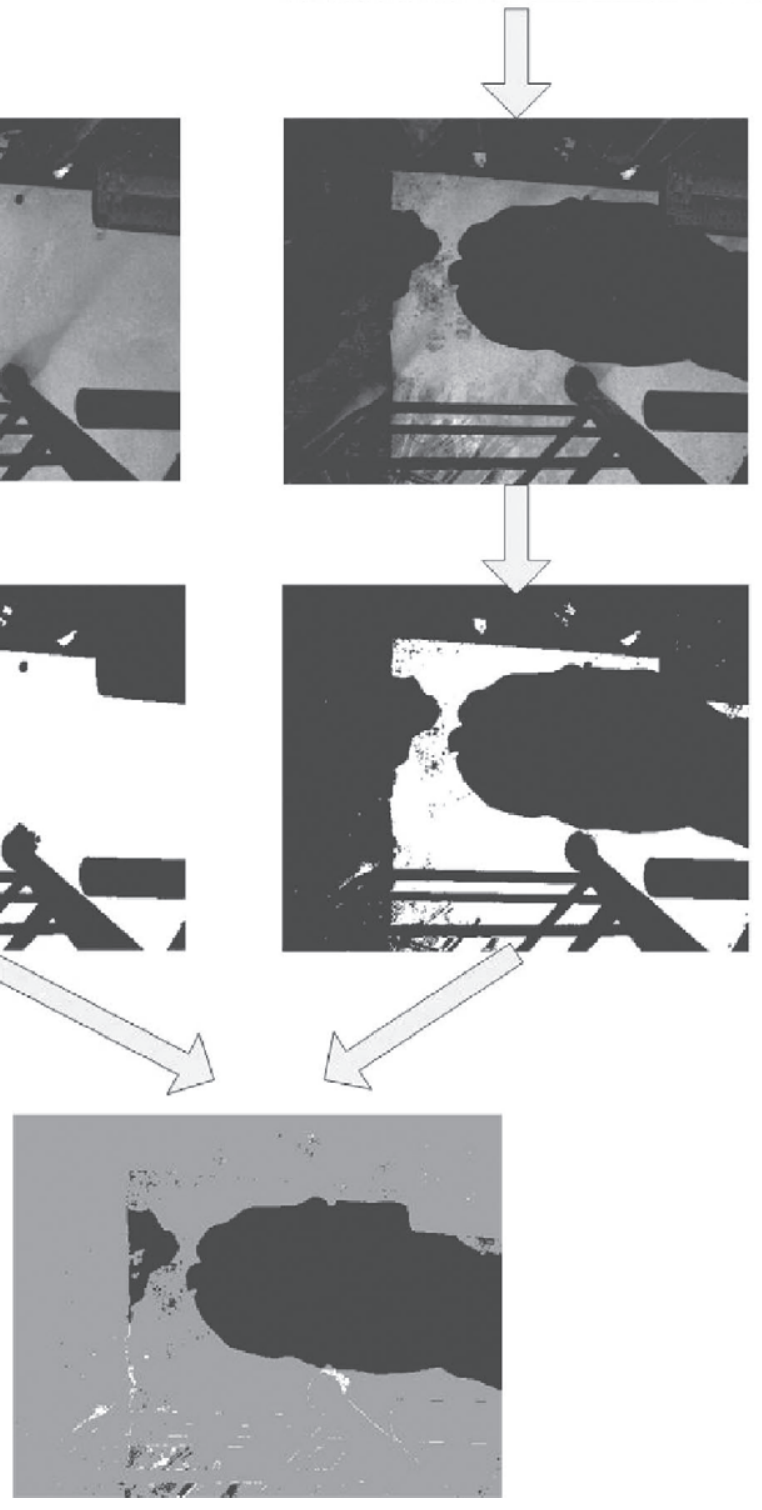
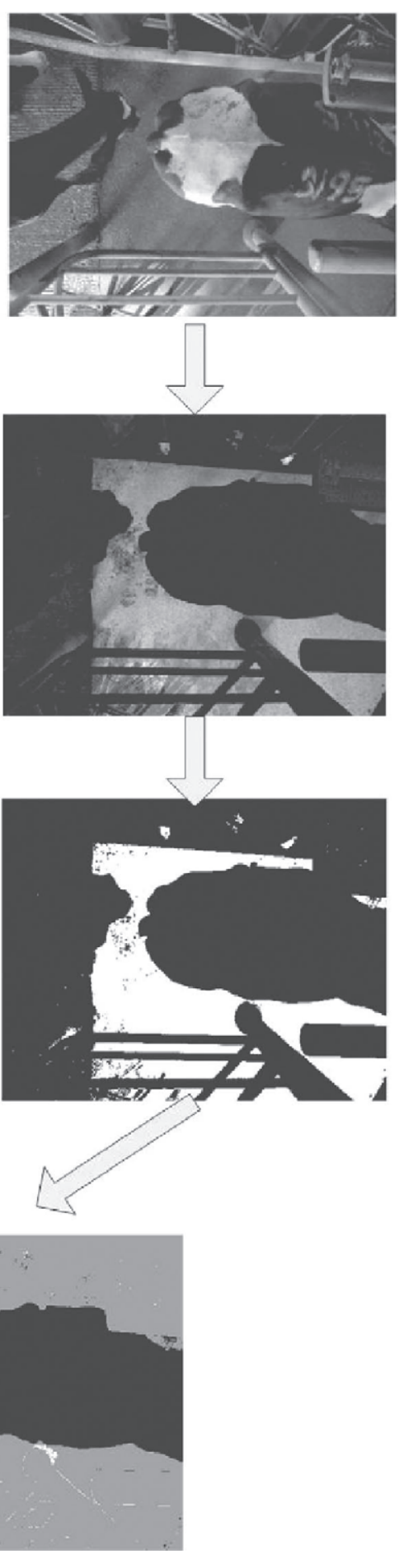

Figure 3. Example of the segmentation procedure for the image of cow number 3195. (a) The original background and cow image; (b) the

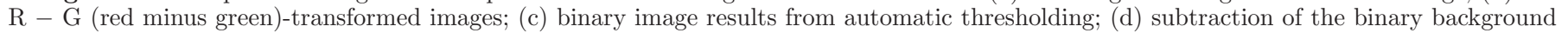
image from binary cow image; (e) removal of noise by saving the largest object in the image; (f) smooth image after erosion and dilation; (g)

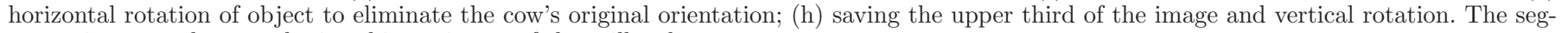
mentation procedure results in a binary image of the tailhead area. e

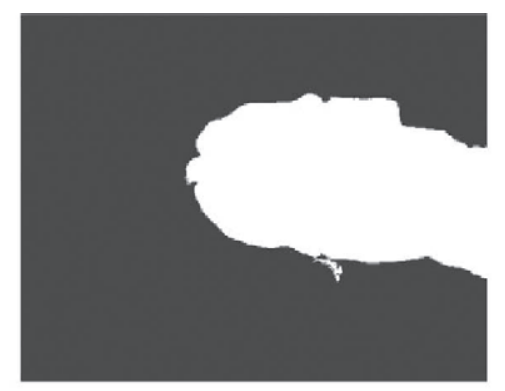

$\mathbf{f}$

g

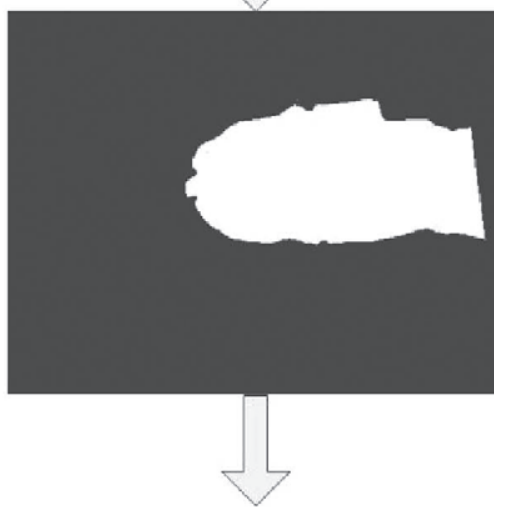

h

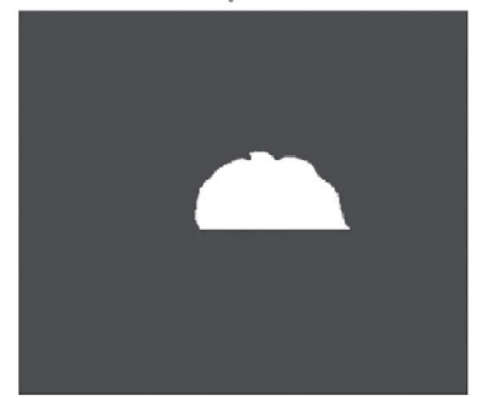



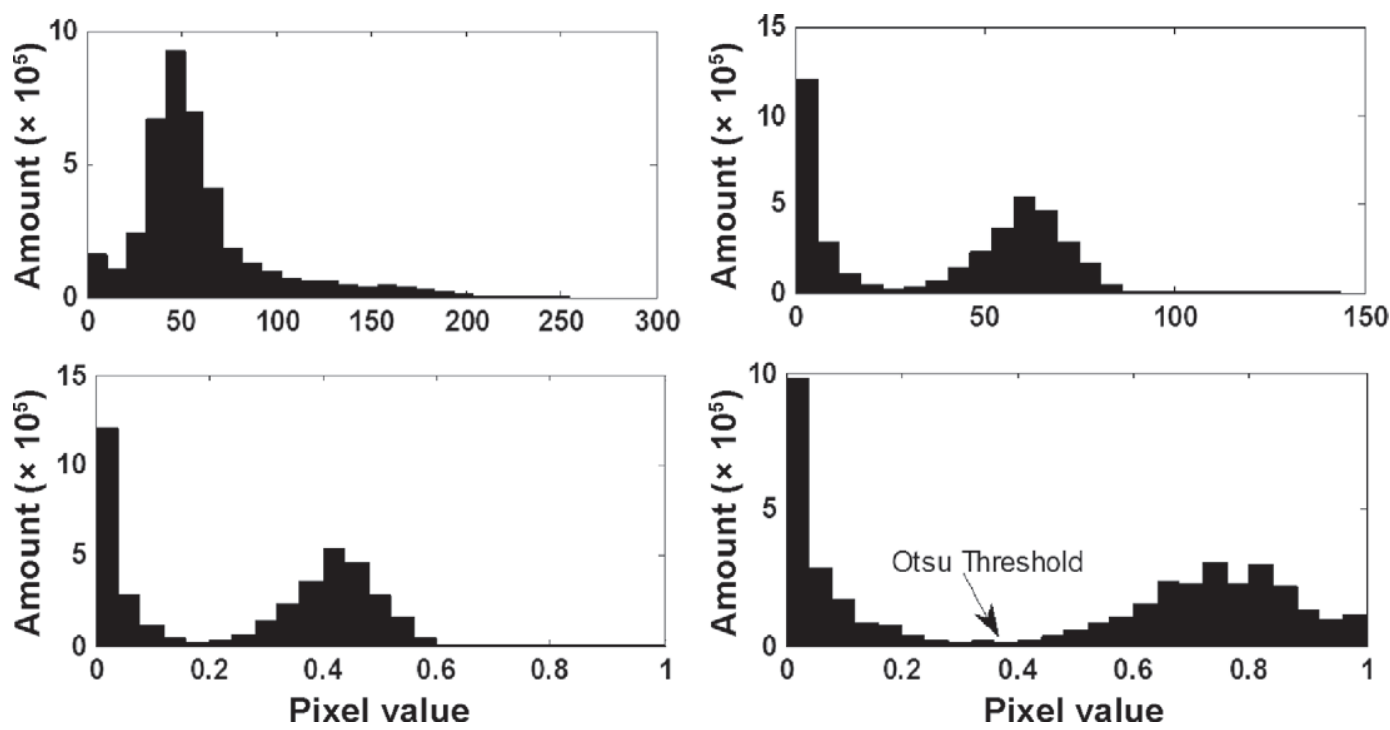

Figure 4. Grayscale pixel value histogram of the background image in the RGB (red-green-blue) space (upper left). Upper right: pixel value distribution of $\mathrm{R}-\mathrm{G}$-transformed image is bimodal. Bottom left: the pixel value histogram is presented in grayscale (values in this histogram are between 0 and 1); bottom right: pixel value distribution after increasing contrast; at this stage the threshold can be computed.

est 1,500 points. Then, interpolation was conducted to include a contour of 1,000 points scaled to a 0:1 range (scaling was performed by calculating the maximum and minimum coordinates for each axis).

Anatomical Point Extraction. The normalized contour was smoothed using the Savitsky-Golay algorithm (PLS toolbox, Eigenvectors Inc., Seattle, WA). This algorithm receives the size of the window (in this case defined as 25 points) and fits the points within the window to a line (the polynomial degree was set to 1). This smoothing allows easy selection of peaks and valleys. The peaks and valleys were selected in the surroundings of 100 points along the contour due to noise that might still appear in the signal. Each one of the desired points was a local extreme point. Their types were maximum point $(\mathrm{n}=9)$, minimum point $(10)$, maximum point (12), minimum point (14), and maximum point (15). This information enabled to detect those points by analyzing the extremes points' type sequence. The vertical and horizontal distances between those points were computed by using their coordinates. To compute the 5 angles, 2 more points were added by constructing a horizontal line from the valley points to the contour line.

Cow Signature Extraction. The cow signature was generated from the normalized contour. As described above, the tailhead contour is a 2-dimensional vector $(x$ and $y$ coordinates). The cow signature was computed by assigning the centroid as $(0.5,0.5)$, and each value in the cow signature is the Euclidean distance between each point in the normalized contour (which is scaled between 1 and 0 ) and the center point. A low-pass filter of moving averages with a window of size of 5 was used to smooth the signature.

\section{Prediction Models}

Three prediction models were developed: the first (model [1]) examined the anatomical points' features by applying multivariable regression. The distances X1 to X5, Y1 to Y5, and Angle1 to Angle5 were the regression predictors (Figure 5).

Models [2] and [3] applied the cow signature. The cow signature vector length was determined with 1,000 values (of Euclidean distances from the contour centroid). Model [2] applied PLS regression (PLSR) to calculate a small number of latent variables that describe most of the variance between the cow's signatures. To avoid over-fitting of the variables selected to the data, the number of latent variables was selected as the number that minimizes root mean square error (RMSE), using LOOM. Model [3] used the absolute values of the first $10 \mathrm{FD}$ of the cow's signatures as prediction variables for the linear regression model. Model [2] was modified with the PLS toolbox. In all models, only significant $(P$ $<0.05)$ variables were incorporated.

\section{Model Evaluation}

All models were developed using the training data set. The best model was validated on a new separate data set - the testing set. The model results were evaluated by $\mathrm{R}^{2}$ and LOOM. Evaluation criteria were (1) actual classification rate; the percentage of correct clas- 


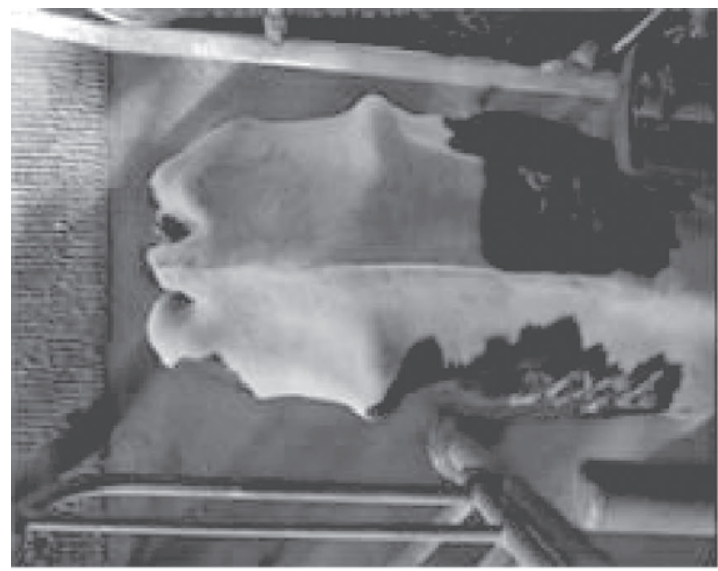

(a)

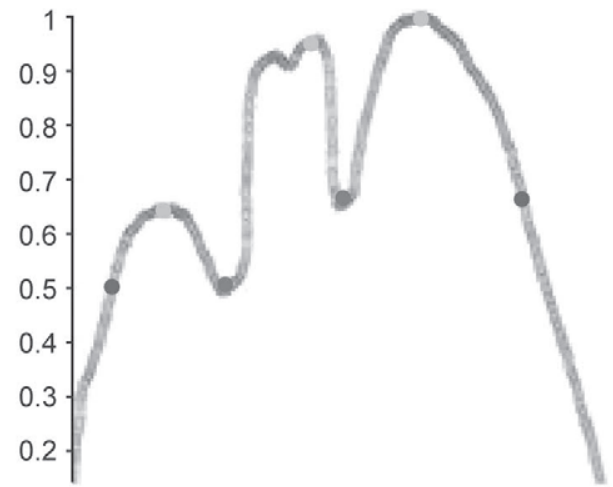

(c)

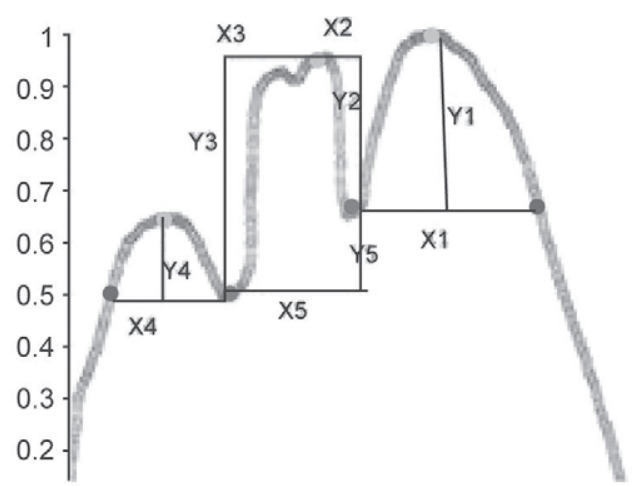

(e)

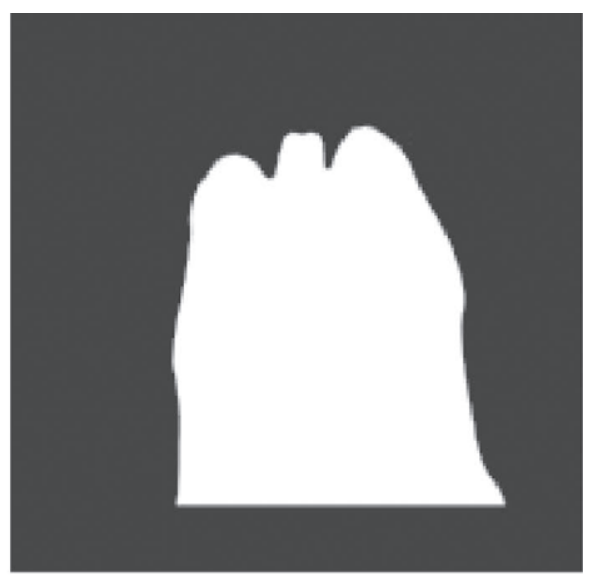

(b)

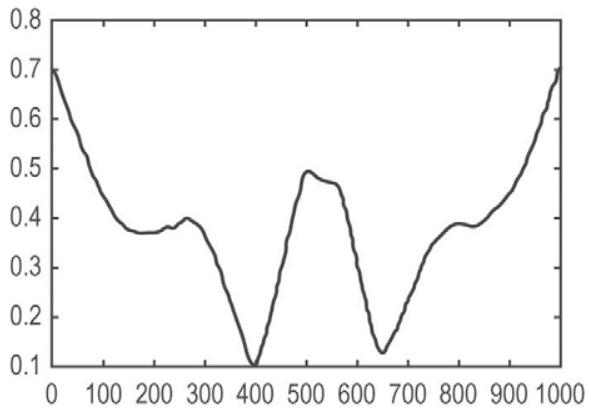

(d)

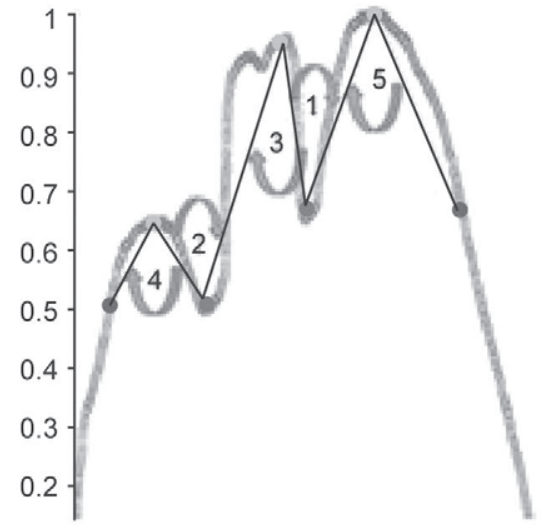

(f)

Figure 5. Example of feature extraction procedure. (a) Original image of cow number 3096; (b) segmentation procedure resulting in binary image of the tailhead area; (c) normalized tailhead contour - the 5 anatomical points were computed on the normalized contour; (d) 1-dimensional cow signature of Euclidean distances of each point in the contour to the object centroid - the cow signature used for BCS prediction in models 2 and 3; (e) vertical and horizontal distances between the 5 anatomical points; and (f) angles between the 5 anatomical points - the distances and angles were used for BCS prediction in model 1 .

sification, within ranges of $0.25,0.5,0.75$, and $1 ;(2)$ the average error rate - the average of the absolute value of the difference between the model score and the manual score; (3) the model's ability to distinguish between
BCS classes, defined as follows: thin cows $(<2.5)$, medium thin (2.5-3), medium fat (3-3.5), and fat (>3.5). Confusion matrices (Fawcett, 2006) were calculated for the different classes. 
Table 2. Correlations between the 5 anatomical-point features and manual scoring (BCS $)^{1}$

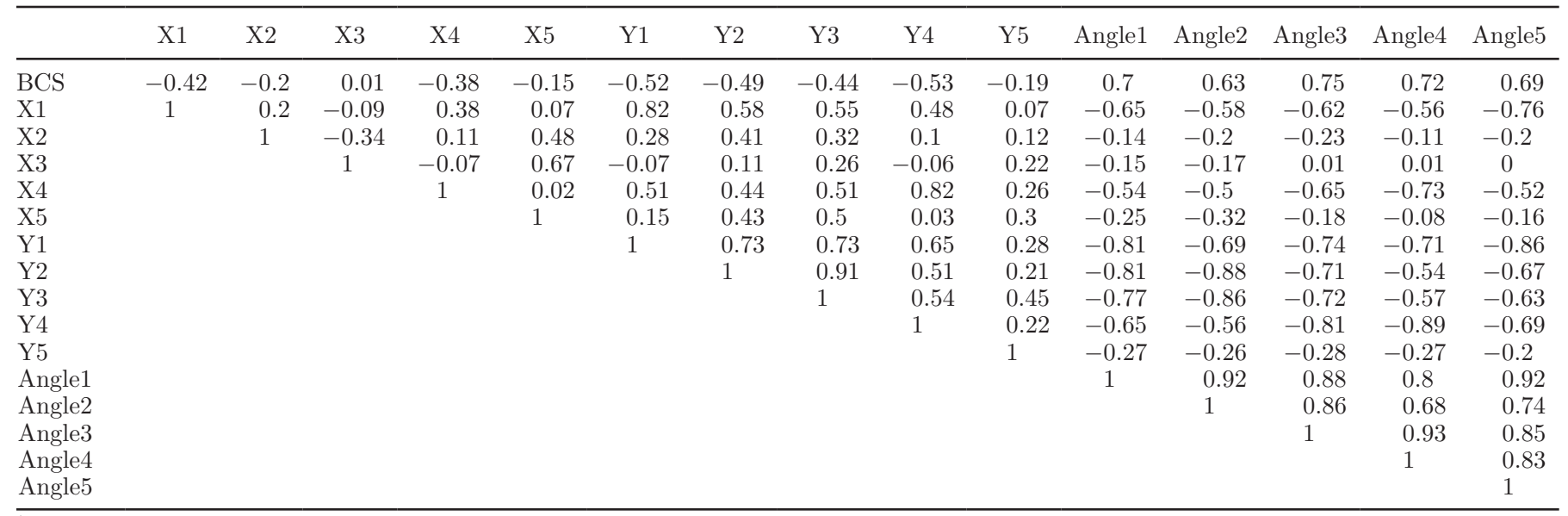

${ }^{1} \mathrm{X} 1 \ldots \mathrm{X} 5=$ horizontal distances (Figure $\left.5 \mathrm{e}\right) ; \mathrm{Y} 1 \ldots \mathrm{Y} 5=$ vertical distances (Figure 5e); Angle1... Angle5 = angles combining the points (Figure $5 f)$.

\section{RESULTS}

\section{Anatomical Point Features (Model [1])}

The linear correlations between the 5 anatomical

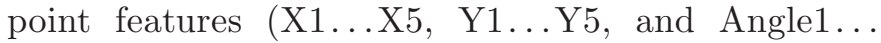
Angle5) and manually determined BCS are shown in Table 2. The Y1, Y4, and Angle1 to 5 features were relatively more correlated $(>0.5)$ with BCS, confirming that the curvature of the tailhead contour reflects BCS: the higher the distance between the peaks and valleys, the thinner the cow (negative correlation), and wide angles indicated a rounder shaped cow (positive correlation). However, Y1 to Y5 and Angle1 to Angle5 features were more interrelated (correlated among themselves), which might limit the model's accuracy.

The $\mathrm{R}^{2}$ obtained by the shape features with the linear regression model was 0.605 . The $\mathrm{R}^{2}$ obtained by LOOM was 0.56 . The obtained model was

$$
\begin{gathered}
\mathrm{BCS}=-0.879+0.015 \operatorname{Avg}(\text { Angle1,2) } \\
+0.02 \operatorname{Avg}(\text { Angle4,5 })+5.745 \operatorname{Avg}(\mathrm{Y} 1,4),
\end{gathered}
$$

where BCS is the computed score of the cow, $\operatorname{Avg}($ Angle1,2) is the average value of Angle1 and Angle2, $\operatorname{Avg}($ Angle4,5) is the average value of Angle4 and Angle5, and $\operatorname{Avg}(\mathrm{Y} 1,4)$ is the average value of $\mathrm{Y} 1$ and Y4. All horizontal measurements (X1 to X5), Y2, Y3, Y5, and Angle3 were excluded from the model. The angles were given in radians and the distances were computed on a scale of 0 to 1 because of the previously discussed contour normalization. All angles and distances are illustrated in Figure 5e and $\mathrm{f}$.

\section{D Cow Signature—PLSR (Model [2])}

The minimum value of RMSE in LOOM was 0.5 when selecting the first 3 latent variables (the RMSE for the calibration was 0.45 ). By selecting more latent variables, the RMSE for the calibration set dropped monotonically, but the RMSE of the LOOM increased, and therefore the number of variables selected was 3 . The $\mathrm{R}^{2}$ with the manual scoring achieved by this model on the training set was 0.6 , and 0.48 using the LOOM, indicating that this method, in this case, does not provide a suitable solution.

Figure 6 shows the signature of 3 thin (broken lines, BCS $<2.5$ ) and 3 fat (solid lines, BCS $>4$ ) cows. It is possible to distinguish the low valleys of the broken lines, but it is also possible to distinguish the variance in their location, making identification difficult with this method.

\section{D Cow Signature-FD (Model [3])}

Figure 7 shows an example of the absolute values of the FD derived from a single cow's signature. The first descriptor was left out of the graph because of its very high value (around 500). It can be seen that the values decrease to almost zero from the 10th descriptor. This indicates that the first $10 \mathrm{FD}$ probably hold most of the information on shape and they were therefore used as prediction variables in model [3]. Figure 8 shows the advantage of using DFT to smooth the curves. Differences can be seen in the 2nd to 10th FD of 4 thin cows $(\mathrm{BCS}<2.5, \mathrm{O})$ and 4 fat cows $(\mathrm{BCS}>4,+)$ : in the 3rd FD, the thin cows have values between 40 and 60 , whereas fat cows have a maximum value of around 30 . In the 5 th FD, thin cows have a value between 20 


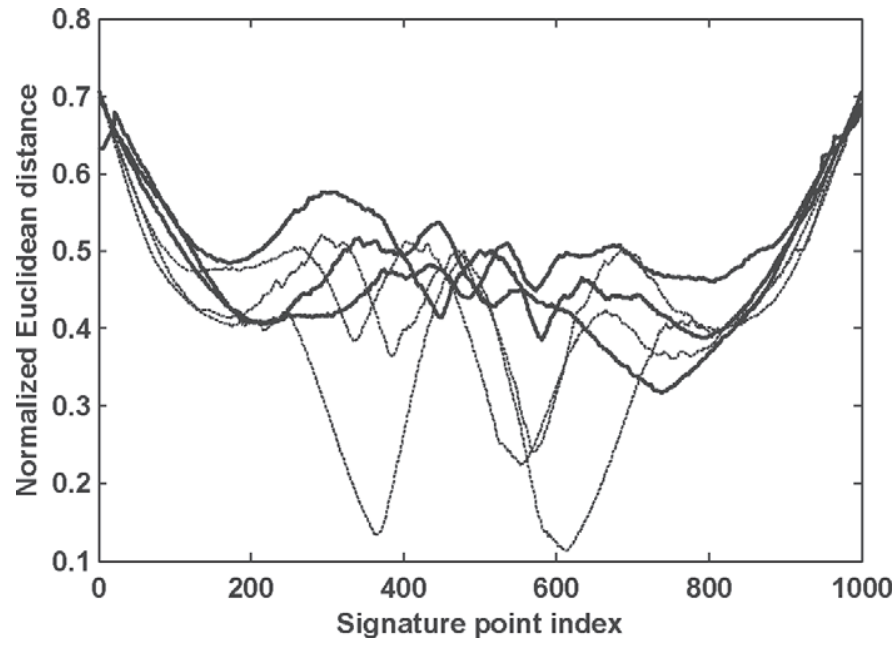

Figure 6. Signature curves of 3 thin cows (thin lines, BCS $<2.5$ ) and 3 fat cows (thick lines, BCS $>4$ ). Each signature is sized at 1,000 points. Note the variance in the locations of the peaks and valleys within the groups.

and 40 , whereas the maximum value of the fat cows is around 15 .

The correlation matrix (Table 3) between manual BCS and FD (F1...F10) indicates that 9 descriptors (the exception was F1) had a negative connection with BCS. In general, no FD had strong correlations (the strongest correlation was achieved by the 5th and 6th FD) with BCS. On the other hand, the FD showed no strong cross-correlation (within FD), indicating that a linear combination between them can be useful. The obtained model was

$$
\begin{aligned}
\mathrm{BCS} & =6.4-0.027 \mathrm{~F} 3-0.024 \mathrm{~F} 4-0.011 \mathrm{~F} 5 \\
& -0.081 \mathrm{~F} 6-0.087 \mathrm{~F} 7-0.072 \mathrm{~F} 8,
\end{aligned}
$$

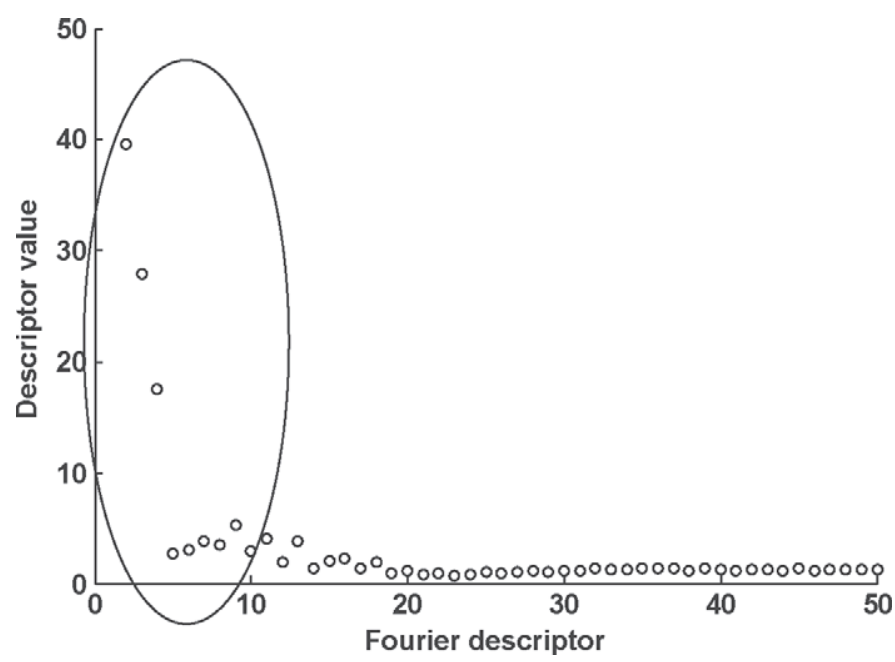

Figure 7. An example of the 2nd to 50th Fourier descriptors of one cow signature. From the 10th descriptor on, the values drop to zero.

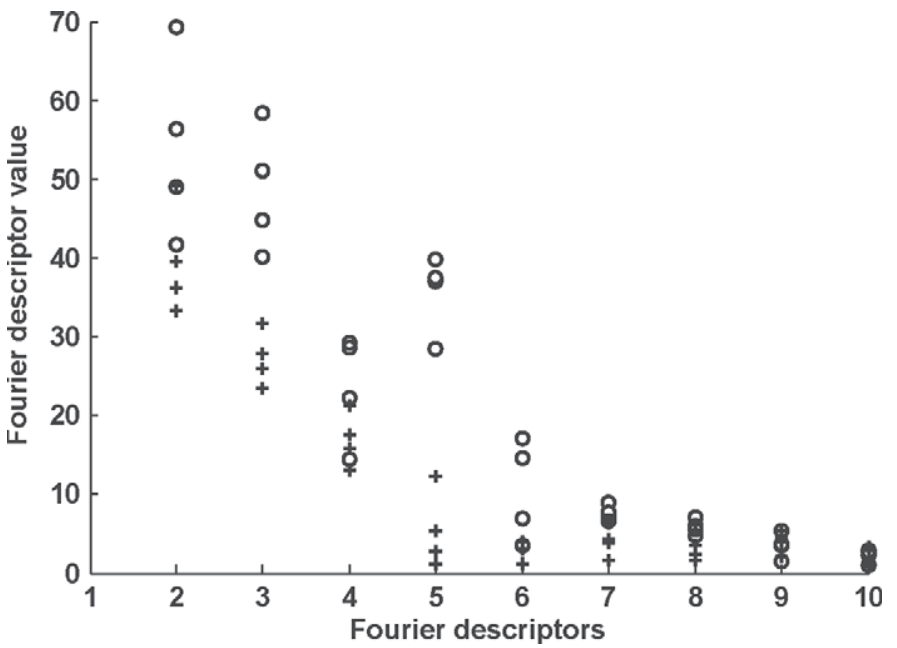

Figure 8. The absolute values of 2 to 10 Fourier descriptors of signatures of 4 thin cows $(\mathrm{O}, \mathrm{BCS}<2.5)$ and 4 fat cows $(+, \mathrm{BCS}>4)$. The difference between the fat group and thin group in this example is particularly noticeable in descriptors 3 and 5 .

where BCS is the cow's computed score, and F3, F4, F5, F6, F7, and F8 are the FD (given in their absolute value) of the specific cow's signature. The $\mathrm{R}^{2}$ obtained by model [3] on the training set were 0.77 and 0.73 for LOOM (Figure 9). In Eq. [2], one can see that for a cow with a straight line (0 frequencies), the score will be 6.4. Although this score and a constant signature (with all FD values being 0) are not possible, it implies that any addition of curvature (frequency) to the cow's contour will directly reduce BCS.

Table 4 summarizes the performance of the 3 models with the best results being obtained for model [3]. Consequently, model [3] was applied to the BCS testing data set.

\section{Performance Measures}

Model performance was validated on the testing data set collected in February 2012. The $\mathrm{R}^{2}$ between the manual BCS and BCS computed by model [3] on the testing set was 0.64 (Figure 10). Actual classification rates of the model on both training and testing sets (Table 5) indicate similar performance, with approximately $50 \%$ of the scores computed by model [3] being within the 0.25 -point range of the manual score, and almost $100 \%$ of the scores being within the range of 0.75 points from the manual score. The average absolute error between the model score and the manual score on the testing set was 0.34 .

In Table 6, 4 classes are presented: thin cows $(<2.5)$, medium-thin (2.5-3), medium-fat (3-3.5), and fat $(>3.5)$. In the training set (Table 6 ), only 1 cow was misclassified by more than one class (classified as 2.5-3 instead of 3.5-5) and in the testing set (Table 6), 2 cows 
Table 3. Correlations between the first 10 Fourier descriptors (F1 to F10) and manual scoring (BCS)

\begin{tabular}{|c|c|c|c|c|c|c|c|c|c|c|}
\hline & F1 & F2 & F3 & F4 & F5 & F6 & F7 & F8 & F9 & F10 \\
\hline BCS & 0.47 & -0.15 & -0.09 & -0.39 & -0.52 & -0.59 & -0.35 & -0.30 & -0.11 & -0.02 \\
\hline F1 & 1.00 & -0.77 & -0.79 & -0.19 & -0.81 & -0.13 & 0.39 & -0.06 & -0.10 & -0.10 \\
\hline $\mathrm{F} 2$ & & 1.00 & 0.65 & 0.16 & 0.60 & -0.01 & -0.49 & -0.08 & 0.05 & 0.07 \\
\hline F3 & & & 1.00 & -0.03 & 0.36 & -0.35 & -0.59 & 0.08 & 0.05 & 0.01 \\
\hline F4 & & & & 1.00 & 0.15 & 0.06 & -0.01 & 0.39 & 0.05 & -0.28 \\
\hline F5 & & & & & 1.00 & 0.47 & -0.21 & -0.03 & 0.12 & 0.26 \\
\hline F6 & & & & & & 1.00 & 0.38 & -0.15 & 0.09 & 0.21 \\
\hline F7 & & & & & & & 1.00 & 0.04 & -0.27 & -0.14 \\
\hline F8 & & & & & & & & 1.00 & 0.24 & -0.21 \\
\hline F9 & & & & & & & & & 1.00 & 0.25 \\
\hline F10 & & & & & & & & & & 1.00 \\
\hline
\end{tabular}

were misclassified by more than one class (classified as $1-2.5$ instead of 3-3.5 and classified as $2.5-3$ instead of $3.5-5)$. The weakness of the model's performance can be noted in the confusion matrices (Table 6) in the model's output of class 2.5-3, where the cow's actual class according to manual classification comes from all 4 existing classes ( $48 \%$ and $52 \%$ of cows that were classified as $2.5-3$ in the training and testing respectively, were actually misclassified: column 2 in Table 6). In addition, in the testing set (Table 6), the problem of classifying fat cows is more obvious, where most fat cows $(>3.5)$ were classified as 3-3.5.

The model's repeatability was measured in 5 images of 5 different cows. Table 7 shows the BCS and its standard deviation. The largest deviation was 0.21 , less

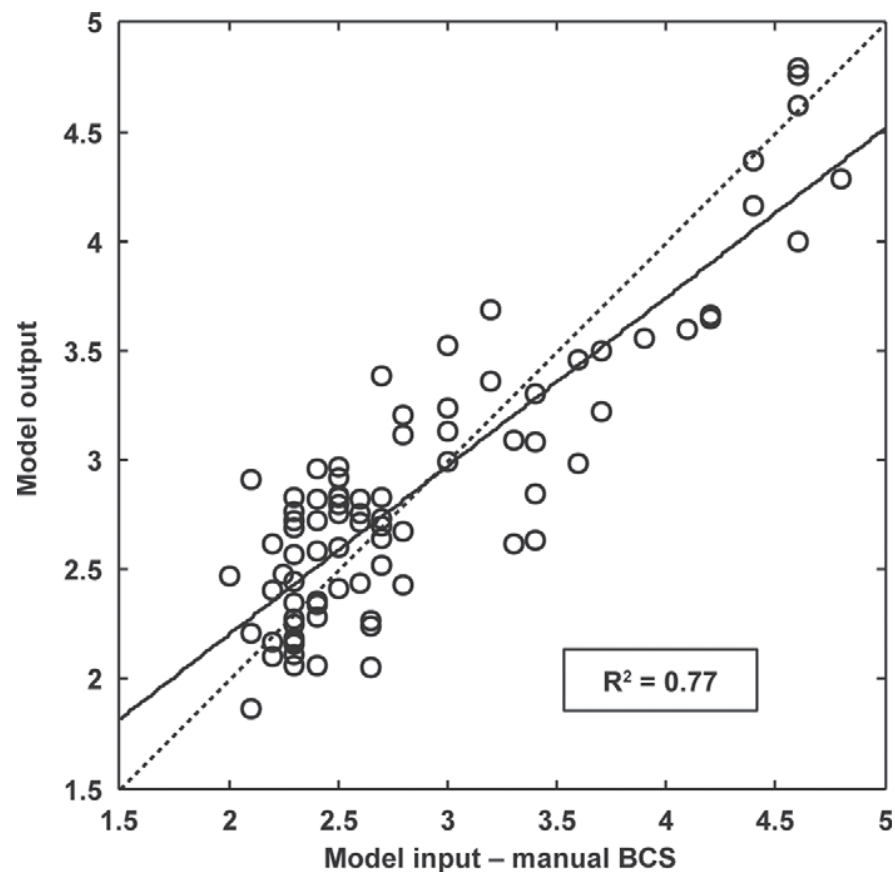

Figure 9. Correlation in training set between the model output (model 3, y-axis) and manual scoring (x-axis) of BCS. The solid line is the linear fit line and the broken line is the 1:1 line. than 1 digression on the Ferguson scale (0.25). Figure 11 shows an evaluation of the model's repeatability.

\section{DISCUSSION}

The combination of anatomical points with FD in one model did not improve results. The anatomical points features (in backward, stepwise, or forward methods) seemed to be excluded from the model. Applying PLSR on the entire set of FD (1,000 descriptors) also did not improve results, implying that the Fourier method is preferred. The FD are not influenced by the variance in peak and valley locations, and they allow clear and easy reduction of the problem dimension by describing the data with only $1 \%$ of the descriptors. In

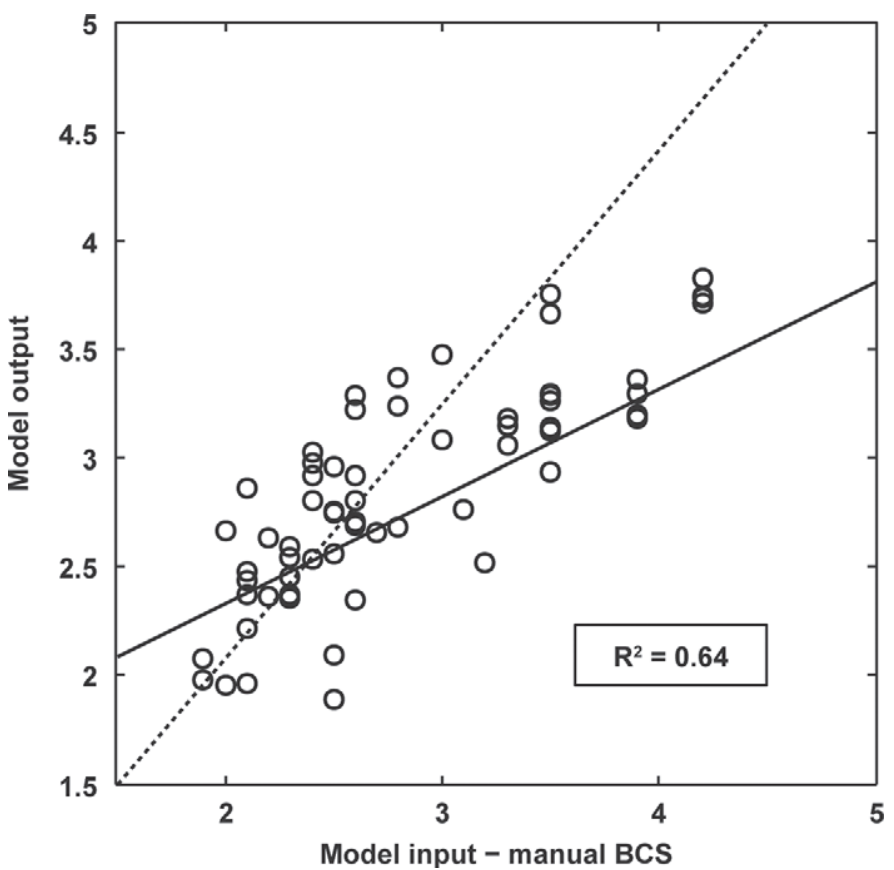

Figure 10. Correlation in testing set between the model output (model 3, y-axis) and manual scoring (x-axis) of BCS. The solid line is the linear fit line and the broken line is the 1:1 line. 
Table 4. The linear regression results of the modified models ${ }^{1}$

\begin{tabular}{|c|c|c|c|}
\hline Model & Feature & $\mathrm{R}^{2}$ & $\begin{array}{c}\mathrm{R}^{2} \\
(\mathrm{LOOM})\end{array}$ \\
\hline 1 & Five anatomical point features & $0.65^{* *}$ & $0.56^{* *}$ \\
\hline 2 & Three latent variable cow signatures & $0.60^{* *}$ & $0.41^{* *}$ \\
\hline 3 & First 10 Fourier descriptors of cow signatures & $0.77^{* *}$ & $0.73^{* *}$ \\
\hline
\end{tabular}

addition, the FD are less sensitive to noise, and they allow smoothing of the signature by usage of a lowpass filter and placing the noise in the high frequencies (Zhang and Lu, 2004).

Several methods have been used in previous research to evaluate BCS model performance: average error rate (Azzaro et al., 2011), $\mathrm{R}^{2}$ (Halachmi et al., 2008), and actual classification results (Bewley et al., 2008). The average error rate tested by Azzaro et al. (2011) was 0.31 (for the PCA model of 23 anatomical points). The model developed in our research gave an average error rate of 0.34 on the testing set. However, the method of Azzaro et al. (2011) involved a manual labeling step. The model developed in our research showed weaker results in terms of $\mathrm{R}^{2}(0.77$ vs. 0.89$)$ than the parabolic model (Halachmi et al., 2009). However, the latter model (Halachmi et al., 2008) was established using a thermal camera. This camera allows better segmentation of the entire body of the cow (including the hook area) but is expensive. Bewley et al. (2008) showed that a model based on the angles of 23 anatomical points correctly classifies $100 \%$ of the scores within the 0.5 range and $93 \%$ of them within the 0.25 range. In the current proposed method, about half of the observations were

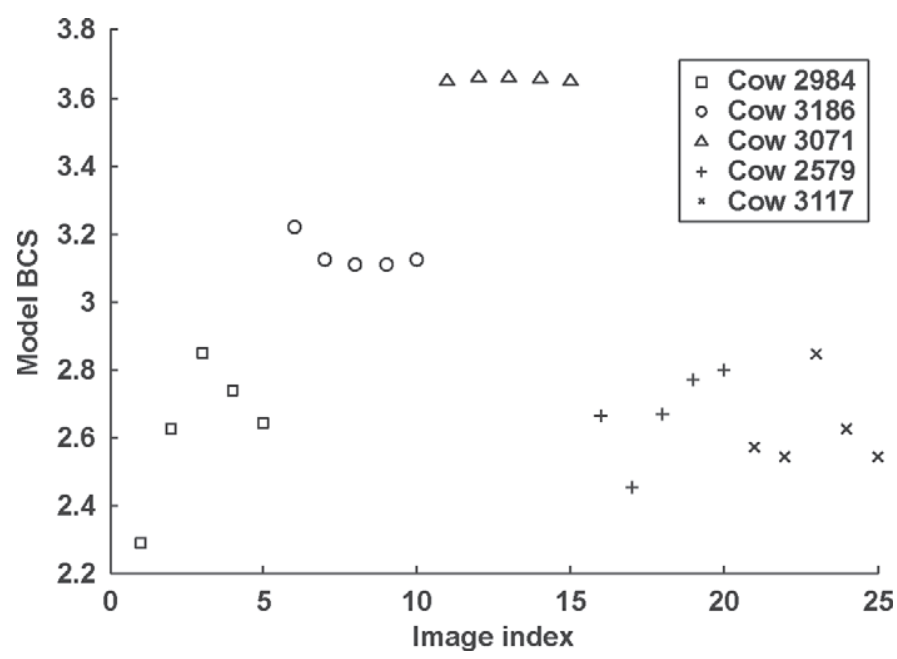

Figure 11. Body condition score computed for 5 different images of 5 different cows taken on the same day. misclassified within the range of 0.25 (58\% in testing set), and $82 \%$ were classified correctly within the 0.5 range (in the testing set, $72 \%$ ). Potential reasons for the weaker results are that the method of Bewley et al. (2008) involved manual labeling and used the hook area in addition to the tailhead; the hook area was impossible to extract automatically under our research conditions. In general, these results imply that the proposed model is as good as state-of-the-art models; however, its main advantage is that it does not require any manual labeling of points in the cow image or manual interference in the segmentation procedure and it can be implemented with a low-cost digital camera.

We proposed the confusion matrix as an additional performance measure because it is commonly used in classification problems and also because of (1) the inherent subjectivity of the gold standard, (2) the possibility of large variation in the experts' golden reference, and (3) vague borders between classes. In practice, the main interest is in the BCS class and trend, not the absolute numerical value. In other words, it is important to know whether a cow is thin, very thin, or fat, but not important to know its specific BCS value (values of 2.4, 2.5, and 2.6. are quite similar). Ferguson et al. (2006) stated that, in general, cows with BCS $<2.5$ are too thin and cows with BCS $>3.5$ are too fat. Therefore, actual classification results were derived for the following classes: thin $(<2.5)$, medium-thin $(2.5-3)$, medium-fat (3-3.5), and fat (>3.5). On the other hand, this type of classification (confusion matrix) is problematic for the following reasons. First, there is high dependency on the values of the borders, which might not be important; if we take, for instance, the 11 cows that were misclassified as 2.5-3 instead of 2-2.5 in the training set (Table 6 ), 6 were actually very close to

Table 5. Classification rates (\%) within different BCS ranges

\begin{tabular}{lcccc}
\hline & \multicolumn{4}{c}{ BCS range } \\
\cline { 2 - 5 } Set & $0-0.25$ & $0-0.5$ & $0-0.75$ & $0-1$ \\
\hline Training set & 53 & 82 & 98 & 100 \\
Testing set & 43 & 72 & 94 & 100 \\
\hline
\end{tabular}


Table 6. Classification results of thin $(<2.5)$, medium thin $(2.5-3)$, medium fat $(3-3.5)$, and fat $(>3.5)$ cows in training and testing data sets

\begin{tabular}{|c|c|c|c|c|c|c|c|c|c|c|}
\hline \multirow{2}{*}{ Real BCS } & \multicolumn{10}{|c|}{ Model output BCS } \\
\hline & \multicolumn{5}{|c|}{ Training data set, no. (\%) } & \multicolumn{5}{|c|}{ Testing data set, no. (\%) } \\
\hline $1.0-2.5$ & $21(66)$ & $11(34)$ & $0(0)$ & $0(0)$ & $32(100)$ & $14(64)$ & $8(36)$ & $0(0)$ & $0(0)$ & $22(100)$ \\
\hline $2.5-3.0$ & $6(23)$ & $17(65)$ & 3 (12) & $0(0)$ & $26(100)$ & $3(18)$ & $10(59)$ & $4(23)$ & $0(0)$ & $17(100)$ \\
\hline $3.0-3.5$ & $0(0)$ & $4(30)$ & $7(55)$ & $2(15)$ & $13(100)$ & $1(14)$ & $2(29)$ & $4(57)$ & $0(15)$ & $7(100)$ \\
\hline
\end{tabular}

Table 7. Repeatability BCS for each cow and its standard deviation (in parentheses in the last row)

\begin{tabular}{ccccc}
\hline Cow 2984 & Cow 3186 & Cow 3071 & Cow 2579 & Cow 3117 \\
\hline 2.29 & 3.22 & 3.65 & 2.67 & 2.57 \\
2.63 & 3.12 & 3.66 & 2.46 & 2.54 \\
2.85 & 3.11 & 3.66 & 2.67 & 2.85 \\
2.74 & 3.11 & 3.66 & 2.77 & 2.62 \\
2.64 & 3.12 & 3.65 & 2.8 & 2.54 \\
$(0.21)$ & $(0.05)$ & $(0.01)$ & $(0.13)$ & $(0.13)$ \\
\hline
\end{tabular}

their real class $(<0.25$ from the class border), with 3 out of those 6 at a distance of approximately 0.1 from their real class. This implies that prediction ability is strong despite the errors in exact class assignment which, as discussed above, is problematic. Second, for practical implementation, analyzing the individual cow might be problematic; for example, a cow could lose 0.49 units of BCS but still be in the same class, which might mask the real status of the cow (her trend), especially in early lactation when losing 0.49 units of BCS is very significant. Therefore, the confusion matrix can also be applied with classes within a 0.25 point range. In addition to identifying trends of individual cows, monitoring the status of a group of cows indicates a nutritional problem for the entire group.

The implementation of this research must deal with several issues related to model calibration, a common procedure for sensory technology implementation. In this study, all segmentation procedures were automatic. However, because the color of the background (the floor) and lighting conditions might differ from barn to barn, a color calibration procedure must be included. The model must refer to a gold standard instead of a single expert as conducted in this study. However, this gold reference must be developed. In a previous study, Halachmi et al. (2008) reported that 2 observers and an ultrasound technician (3 observers in total) estimated the subcutaneous backfat thickness. The gold reference could include calibration on many cows and many observers and define a strict standardization process in many farms. Further research should address BCS for other cow breeds and might require specific calibrations for each breed.
In addition, research is underway to automate the frame-selection procedure and filter out useless images (e.g., cow out of frame, problematic tail orientation). We are investigating the possibility of applying a lowcost 3D camera. Additional training with a larger database might improve results.

\section{CONCLUSIONS}

Presenting the cow's tailhead contour as a set of Euclidean distances from each point in the contour to the object center (cow signature) seems to be an efficient feature for automatic BCS prediction. The first $10 \mathrm{FD}$ held most of the information in the signature, making the possibility of automatic BCS prediction feasible.

\section{ACKNOWLEDGMENTS}

Special thanks go to the anonymous reviewers of the Journal of Dairy Science whose advice and recommendations ensured the high quality of the paper. We thank Shamay Yakobi and the farm workers at the ARO's research farm at the Volcani Center for their help and support in the data collection. This research was funded by the Ministry of Agriculture Chief Scientist Fund, ARO No. 459-4396-10 and partially supported by the Paul Ivanier Center for Robotics Research and Production Management, and the Rabbi W. Gunther Plaut Chair in Manufacturing Engineering, Ben-Gurion University of the Negev, Israel.

\section{REFERENCES}

Azzaro, G., M. Caccamo, J. D. Ferguson, S. Battiato, M. Farinella, C. Guarnera, G. Puglisi, R. Petrigiliero, and G. Licitra. 2011. Objec- 
tive estimation of body condition score by modeling. J. Dairy Sci. 94:2126-2137.

Bewley, J. M., A. M. Peacock, O. Lewis, R. E. Boyce, D. J. Roberts, M. P. Coffey, S. J. Kenyon, and M. M. Schutz. 2008. Potential for estimation of body condition scores in dairy cattle from digital images. J. Dairy Sci. 91:3439-3453.

British Standards Institution. 1975. Precision of test methods. 1: Guide for the determination and reproducibility for a standard test method. BS 597, Part 1. British Standards Institution, London, UK.

Brosnan, T., and D. W. Sun. 2004. Improving quality inspection of food products by computer vision-A review. J. Food Eng. 61:316.

Canny, J. 1986. A computational approach to edge detection. IEEE Trans. Pattern Anal. Mach. Intell. 8:679-698.

Coffey, M. P., T. B. Motram, and N. McFarlance. 2003. A feasibility study on the automatic recording of condition score in dairy cows. Page 131 in Proc. Annu. Mtg. Br. Soc. Anim. Sci., March. BSAS, Penicuik, Midlothian, UK.

Fawcett, T. 2006. An introduction to ROC analysis . Pattern Recognit. Lett. 27:861-874.

Ferguson, J. D., G. Azzaro, and G. Licitra. 2006. Body condition assessment using digital images. J. Dairy Sci. 89:3833-3841.

Ferguson, J. D., D. T. Galigan, and N. Thomsen. 1994. Principal descriptors of body condition score in Holstein cows. J. Dairy Sci. 77:2695-2703.

Hady, P. J., J. J. Domecq, and J. B. Kaneene. 1994. Frequency and precision of body condition scoring in dairy cattle. J. Dairy Sci. $77: 1543-1547$.
Halachmi, I., P. Polak, D. J. Roberts, and M. Klopcic. 2008. Cow body shape and automation of scoring BCS. J. Dairy Sci. 91:4444-4451.

Halachmi, I., P. Polak, D. J. Roberts, M. Klopcic, and J. M. Bewley. 2009. Thermally sensed, automatic, cow body condition scoring. Pages 193-201 in Proc. 4th Eur. Conf. Precision Livestock Farming, Wageningen, the Netherlands. Wageningen Press, Wageningen, the Netherlands.

Heinrichs, A. J., and V. A. Ishler. 1989. Body condition scoring as a tool for dairy herd management. Extension Circular 363. College of Agriculture Cooperative Extension, University of Pennsylvania, University Park.

Otsu, N. 1979. A threshold selection method from gray-level histograms. IEEE Trans. Syst. Man Cybernet. 9:62-66.

Roche, J. R., N. C. Friggens, J. K. Kay, M. W. Fisher, K. J. Stafford, and D. P. Berry. 2009. Body condition score and its association with dairy cow productivity, health, and welfare. J. Dairy Sci. 92:5769-5801.

Stone, M. 1974. Cross-validatory choice and assessment of statistical predictions. J. R. Stat. Soc. 36:111-147.

Zhang, D., and G. Lu. 2004. Review of shape representation and description techniques. Pattern Recognit. 37:1-19.

Zion, B., V. Alachantis, V. Ostrovsky, A. Barki, and I. Karplus. 2006. Real-time underwater sorting of edible fish species. Comput. Electron. Agric. 56:34-45.

Ziou, D., and S. Tabbone. 1997. Edge detection techniques-An overview. http://citeseerx.ist.psu.edu/viewdoc/summary?doi= 10.1.1.27.1821. 